\title{
Tannic acid inhibits the Jak2/STAT3 pathway and induces G1/S arrest and mitochondrial apoptosis in YD-38 gingival cancer cells
}

\author{
PRAMOD DARVIN $^{1}$, SEUNG JO BAEG ${ }^{2}$, YOUN HEE JOUNG ${ }^{1}$, NIPIN SP ${ }^{1}$, DONG YOUNG KANG ${ }^{1}$, \\ HYO JOO BYUN ${ }^{1}$, JE UK PARK ${ }^{2}$ and YOUNG MOK YANG ${ }^{1}$ \\ ${ }^{1}$ Department of Pathology, School of Medicine, and Institute of Biomedical Science and Technology, \\ Konkuk University, Seoul 143-701; ${ }^{2}$ Department of Oral and Maxillofacial Surgery, \\ College of Medicine, The Catholic University of Korea, Seoul 137-701, Republic of Korea
}

Received April 13, 2015; Accepted May 18, 2015

DOI: $10.3892 / \mathrm{ijo} .2015 .3098$

\begin{abstract}
Tannic acid (TA), is a potent anti-oxidant, showing anti-proliferative effects on numerous cancers. The ability of TA to induce proliferation inhibition on the rare tumor, gingival squamous cell carcinoma (GSCC), comprising $<10 \%$ of all head and neck squamous cell carcinomas was studied in the YD-38 cell line. The main goal was to modulate the Jak2/STAT3 pathway using TA and to induce cell cycle arrest and apoptosis in GSCC. TA treatment induced G1 arrest and apoptosis in YD-38 cells. Molecular analysis revealed that TA inhibits Jak2/STAT3 pathway by preventing their expression as well as phosphorylation. This inhibition of STAT3 phosphorylation prevented the nuclear translocation and DNA binding capability of STAT3. Together with the inhibition of transcriptional regulatory function of STAT3, TA inhibited the expression of G1 phase modulators CDK-4, CDK-6, cyclin D1 and cyclin $\mathrm{E}$. It is also evidenced that TA exerted an intense activation of $\mathrm{p} 21^{\text {Wafl/Cip1 }}, \mathrm{p} 27^{\mathrm{Kip} 1}$ and $\mathrm{p} 53$ genes confirming its role in G1 phase inhibition. Additionally, upon treatment with $\mathrm{TA}$, the expression of mitochondrial pore factors Bax, Bcl-2 and $\mathrm{Bcl}-\mathrm{X}_{\mathrm{L}}$ were changed. We observed inhibition of Bcl-2 and an increase in mitochondrial localization of Bax leading to the loss of mitochondrial membrane potential, resulting in the release of cytochrome $c$ to the cytosol. In addition, we perceived the activation of caspases upon TA treatment. Specific inhibition of caspase protected the cells from TA
\end{abstract}

Correspondence to: Professor Young Mok Yang, Department of Pathology, School of Medicine, and Institute of Biomedical Science and Technology, Konkuk University, Seoul 143-701, Republic of Korea E-mail: ymyang@kku.ac.kr

Professor Je Uk Park, Department of Oral and Maxillofacial Surgery, College of Medicine, The Catholic University of Korea, Seoul 137-701, Republic of Korea

E-mail: jupark@catholic.ac.kr

Key words: Jak2/STAT3, G1 arrest, intrinsic apoptosis, tannic acid, $\mathrm{p} 21^{\text {Wafl/Cipl }}$ induced apoptosis. Taken together, this study reveals that TA significantly inhibits the Jak2/STAT3 signaling pathway and induces G1 arrest and mitochondrial apoptosis in YD-38 cells.

\section{Introduction}

Gingival squamous cell carcinoma (GSCC) is a rare tumor comprising $<10 \%$ of all head and neck squamous cell carcinomas $(1,2)$. It may occur in either the mandible or maxilla (3). This type of cancer typically resembles common periodontal lesion or inflammatory lesion and usually results in delayed diagnosis. Multiple prognostic factors such as tumor size and lymph node metastasis are associated with GSCC, therefore, tumor-node-metastasis (TNM) classification is used for staging GSCC. Gingival cancer has high risk of metastases and consequent death with bone invasion on high-grade tumors (4). In many case reports, the deaths associated with GSCC is due to delayed diagnosis and treatments.

Tannins are polyphenols of plant origin found in vegetables, fruits, red wine, tea, nuts, beans and coffee. Tannins are grouped into two major categories as hydrolysable and condensed tannins. Commercially available tannic acid (TA) includes multiple gallotannins with galloyl esters. Tea and red wine are rich source of hydrolysable TA (5). TA shows anticancer activities and cancer protection activities against a broad spectrum of cancers, including chemically induced cancers (6-11).

Evidence suggests that, Janus kinase 2/signal transducer and activator of transcription 3 (Jak2/STAT3) signaling pathways are associated with oncogenesis, progression and metastasis of different cancers. Constitutively active STAT3 is also observed in various malignant transformations in breast (12), head and neck (13), skin (14), ovarian (15), brain (16) and prostrate (17). Tannins and TA containing foods are known to have anticancer activities against breast cancer through modulating the Jak/STAT pathway (18). We have reported that STAT3 modulates VEGF expression through HIF-1 $\alpha$. Similarly our studies with MSM show that, inhibition of Jak2/STAT3 pathway can restrict breast tumor growth and pulmonary metastasis (19). It is also proven that TA has the ability to inhibit the EGF-receptor (20). STAT3 has the direct transcription control over many genes including survivin 
(proliferation), VEGF (angiogenesis), cyclin D1 (cell cycle), $\mathrm{Bcl}-\mathrm{X}_{\mathrm{L}}$ (apoptosis). Hence, the inhibition of STAT3 should lead to induction of apoptosis.

In the present study, we explore the role of TA in modulating the Jak2/STAT3 pathway. We hypothesize that TA induces proliferation inhibition and G1 phase inhibition in gingival cancer cells. In addition, we hypothesized that TA can modulate multiple molecular targets directly related to the mitochondrial apoptotic pathway and induces intrinsic apoptosis.

\section{Materials and methods}

Antibodies and reagents. Roswell Park Memorial Institute medium-1640 (RPMI-1640), 10\% fetal bovine serum (FBS) and trypsin-EDTA were from Gibco-BRL (Grand Island, NY, USA). Jak2, p-Jak2 (Y1007/1008), p-STAT3 (Y705) and p-STAT3 (S727) antibodies were from Cell Signaling (Cell Signaling Technologies, MA, USA). STAT3, Bax, Bcl-2, Bcl- $X_{\mathrm{L}}$, TATA binding protein (TBP), Caspase-3, cytochrome $c, \beta$-actin antibodies and secondary antibody (rabbit, goat anti-mouse IgG-horseradish peroxidase) were obtained from Santa Cruz Biotechnology (Santa Cruz, CA, USA). The enhanced chemiluminescence plus (ECL Plus) detection kit, RT-PCR Premix kits, oligo(dT), Bcl-2, Bcl- $\mathrm{X}_{\mathrm{L}}$, Bax and 18S primer for RT-PCR were from Bioneer (Daejeon, Korea). $\mathrm{DiOC}_{6}$ was from Sigma (St. Louis, MO, USA). Mitochondria isolation kit and Coomassie (Bradford) protein assay kit were from Thermo Scientific (Thermo Scientific, MA, USA). Restore $^{\mathrm{TM}}$ Western Blot Stripping Buffer and NE-PER kits were from Pierce (Rockford, IL, USA). RNeasy mini kit, and the Qiaprep spin miniprep kits were from Qiagen (Hilden, Germany). The electrophoretic mobility shift assay (EMSA) kit and oligonucleotide probes (STAT3) were from Panomics (Redwood City, CA, USA). Vybrant FAM poly-caspases assay kit was from Molecular probes (Eugene, OR, USA) and CaspGLOW $^{\mathrm{TM}}$ fluorescein active caspase-3 staining kit was from eBioscience (San Diego, CA, USA).

Cell culture and maintenance. YD-38 cell lines were cultured and maintained in RPMI-1640 medium containing 10\% serum and $1 \%$ penicillin/streptavidine, respectively. Unless otherwise specified, cells were grown in 10 -cm dishes to $~ 80 \%$ confluence before being placed in serum-free media for 18-24 h. Serumdeprived cells were treated as specified in the figure legends.

Cell proliferation studies using crystal violet assay. Cell proliferation was analyzed using the crystal violet assay. The YD-38 cells were seeded on to 6-well plates and incubated overnight at ambient condition. After 24-h incubation, the cells were treated with increasing concentration of TA $(20-100 \mu \mathrm{M})$ for 24 or $48 \mathrm{~h}$. The cells were washed with PBS and incubated with crystal violet. Excess amount of crystal violet was washed off with water and the dye captured by the cells were dissolved using $1 \%$ SDS. The final colour formed was measured colorimetrically at $570 \mathrm{~nm}$.

Cell cycle analysis. The DNA content of TA or other chemical combinations treated and non-treated YD-38 cells were determined by BD Cycletest Plus DNA reagent kit (BD Biosciences, CA, USA) following the manufacturer's protocol. Briefly, $\sim 5 \times 10^{5}$ cells were induced, or not induced with TA or other chemical combinations for indicated time. The cells were separated, washed twice with PBS and permeabilized using trypsin buffer. The RNA interaction with PI was neutralized by treating the cells with trypsin inhibitor and RNase buffer. These samples were then stained with propidium iodide for $30 \mathrm{~min}$ in the dark and analyzed using FACSCalibur (BD FACSCalibur, CA, USA).

Measurement of apoptosis. Fluorescein-conjugated Annexin V (Annexin V-FITC) was used to quantify the percentage of cells undergoing apoptosis. The necrotic cells were counter stained with propidium iodide (PI). The cells treated or not were washed twice with cold PBS and resuspended in the binding buffer at a concentration of $1 \times 10^{6}$ cells $/ \mathrm{ml}$. Five microliters each of Annexin V-FITC and PI were added to the cell suspension. After incubation at room temperature in the dark for $15 \mathrm{~min}$, the percentages of apoptotic cells were analyzed by flow cytometry (BD FACSCalibur). Cells treated with $10 \mu \mathrm{M}$ camptothecin served as positive control.

Western blotting. The YD-38 cells were treated with TA for determined times and lysed on ice with radioimmunoprecipitation assay (RIPA) lysis buffer, containing $1 \mathrm{X}$ BD baculogold protease inhibitor cocktail (BD Bioscience) and 1X PhosSTOP phosphatase inhibitors (Roche, NJ, USA). Protein concentrations were detected using the Bradford method. Equal amounts of protein obtained by total lysis were separated using SDS-PAGE and blotted onto a nitrocellulose membrane. Blocking was done with either 5\% skim milk or BSA in TBS-T buffer. The membranes were probed with primary antibodies followed by specific HRP conjugated secondary antibodies. Antibody detection was done by using enhanced chemiluminescence (ECL) plus detection kit.

Semi-quantitative reverse transcription polymerase chain reaction $(R T-P C R)$. Total RNA from YD-38 cells were prepared using RNeasy Mini kit (Quiagen, CA, USA) according to the manufacturer's instructions. Equal amount of RNA from each sample reverse transcribed using AccuPower RT-premix kit (Bioneer, Korea) and oligo(dT) primers. PCR was performed using $2 \mu \mathrm{l}$ of the reverse transcription product. The PCR reactions were performed in $25-30$ cycles of denaturation $94-95^{\circ} \mathrm{C}$, annealing $56-60^{\circ} \mathrm{C}$ and an extension of $72^{\circ} \mathrm{C}$. The primers used for the amplification are listed in the Table I. After amplification, the products were visualized in $1.2 \%$ agarose containing ethidium bromide.

Electrophoretic mobility shift assay (EMSA). STAT3 DNA binding activity was detected using EMSA (19). Gingival cancer cells were grown to $\sim 80 \%$ confluence and nuclear protein extracts were prepared using the Nuclear Extraction kit (Affymetrix, CA, USA). EMSA was performed with EMSA gel shift kit (Panomics) according to the manufacturer's protocol. Briefly, the nuclear proteins prepared were subjected for hybridization with a double-stranded, biotin-labeled oligonucleotide probe containing the consensus-binding site for STAT3 (sense strand, 5'-CATGTTATGCATATTCCTGTAAGTG-3'). The protein-DNA complexes were resolved in a $6 \%$ non-denaturing PAGE gel and transferred to Pall Biodyne B nylon membrane 
Table I. RT-PCR primers sequences used for the amplification of multiple human cDNAs.

\begin{tabular}{|c|c|c|c|c|}
\hline Sl no. & Genes & Annealing temperature $\left({ }^{\circ} \mathrm{C}\right)$ & Product size (bp) & Sequences $\left(5^{\prime}-3^{\prime}\right)$ \\
\hline 1 & Cyclin D1 & 58 & 135 & $\begin{array}{l}\text { F-gctgcgaagtggaaaccatc } \\
\text { R-cctccttctgcacacatttgaa }\end{array}$ \\
\hline 2 & CDK-4 & 58 & 541 & $\begin{array}{l}\text { F-ctgagaatggctacctctcgatatg } \\
\text { R-agagtgtaacaaccacgggtgtaag }\end{array}$ \\
\hline 3 & CDK-6 & 58 & 406 & $\begin{array}{l}\text { F-ccgagtagtgcatcgcgatctaa } \\
\text { R-ctttgcctagttcatcgatatc }\end{array}$ \\
\hline 4 & $18 \mathrm{~S}$ & 58 & 490 & $\begin{array}{l}\text { F-cggctaccacatccaaggaa } \\
\text { R-ccggcgtccctcttaatc }\end{array}$ \\
\hline 5 & $\mathrm{P} 16^{\mathrm{Ink} 4}$ & 57 & 138 & $\begin{array}{l}\text { F-agccttcggctgactggctgg } \\
\text { R-ctgcccatcatcatgacctgg }\end{array}$ \\
\hline 6 & $\mathrm{p} 21^{\text {Waf1/Cip1 }}$ & 58 & 494 & $\begin{array}{l}\text { F-ttagggcttcctcctggaggagat } \\
\text { R-atgtcagaaccggctggggatgtc }\end{array}$ \\
\hline 7 & $\mathrm{p} 27^{\mathrm{Kip} 1}$ & 59 & 428 & $\begin{array}{l}\text { F-cctcttcggcccggtggac } \\
\text { R-tttggggaaccgtctgaaac }\end{array}$ \\
\hline 8 & Bax & 58 & 155 & $\begin{array}{l}\text { F-cccgagaggtctttttccgag } \\
\text { R-ccagcccatgatggttctgat }\end{array}$ \\
\hline 9 & Bcl-2 & 60 & 459 & $\begin{array}{l}\text { F-ggtgccacctgtggtccacctg } \\
\text { R-ggtgccacctgtggtccacctg }\end{array}$ \\
\hline 10 & Bcl-X ${ }_{L}$ & 60 & 780 & $\begin{array}{l}\text { F-ttggacaatggactggttga } \\
\text { R-gtagagtggatggtcagtg }\end{array}$ \\
\hline
\end{tabular}

(Pall Life Sciences, NY, USA) and detected using streptavidinHRP and a chemiluminescent substrate.

Measurement of mitochondrial membrane potential $(\Delta \Psi m)$. Changes of mitochondrial membrane potential $(\Delta \Psi \mathrm{m})$ were measured by $\mathrm{DiOC}_{6}$ staining method. Briefly, gingival cancer cell lines treated or not treated with TA were washed and suspended in $0.1 \mu \mathrm{M} \mathrm{DiOC}_{6}$ solution. Cells were then incubated at $37^{\circ} \mathrm{C}$ for 20 min and washed with pre-warmed DPBS and analysed using FACSCalibur.

Isolation of mitochondria. Mitochrondria from TA-treated and non-treated cells were isolated using mitochondria isolation kit (Thermo scientific, USA) following the manufacturer's protocol. Briefly, $2 \times 10^{6}$ cells were treated with mitochondria isolation reagent and incubated on ice. Following incubation, Reagent $\mathrm{B}$ and $\mathrm{C}$ were added with mixing and incubation on ice between each addition. The mixture was centrifuged at $700 \mathrm{x} \mathrm{g}$ for $10 \mathrm{~min}$ and the supernatant was collected and re-centrifuged. The supernatant was collected as the cytosol and the mitochondrial pellet obtained was washed with Reagent $\mathrm{C}$ and used for downstream applications.

Poly-caspase assay. Activation of caspases were studied using Vybrant FAM poly-caspase assay kit, following the manufacturer's protocol. Briefly, gingival cancer cells treated or non-treated with TA or other chemical combinations were suspended at a concentration of $1 \times 10^{6}$ cells $/ \mathrm{ml}$ culture media.
From this $300 \mu 1$ was mixed with $10 \mu 130 \mathrm{X}$ FLICA and incubated $1 \mathrm{~h}$ at $37^{\circ} \mathrm{C}$ and $5 \% \mathrm{CO}_{2}$. Following this, the cells were washed multiple times using $1 \mathrm{X}$ wash buffer. The cells were then analyzed using FACSCalibur.

Active caspase-3 analysis. Caspase-3 activation studies using CaspGLOW $^{\text {тм }}$ fluorescein active caspase-3 staining kit, following the manufacturer's protocol. Apoptosis is induced using $60 \mu \mathrm{M}$ TA. In order to confirm the role of caspase-3 in TA mediated apoptosis, the cells were pre-treated with Z-VAD-FMK. Following this, $300 \mu \mathrm{l}$ of cell suspension containing $1 \times 10^{6}$ cells $/ \mathrm{ml}$ was made and active caspases- 3 stained using FITC-DEVD-FMK. The mixture was incubated at $37^{\circ} \mathrm{C}$ with $5 \% \mathrm{CO}_{2}$ for $1 \mathrm{~h}$. Then the cells were washed with $1 \mathrm{X}$ wash buffer and subjected for FACS analysis.

Statistical analysis. All experiments were repeated at least three times and the results expressed as mean \pm SEM. Statistical analysis was performed with ANOVA and Student's t-test of SAS 9.3 program. One-way analysis of variance (ANOVA) was performed with Duncan's multiple range test. $\mathrm{P}<0.05$ was considered statistically significant.

\section{Results}

Tannic acid induces proliferation inhibition in gingival cancer cells. The ability of tannic acid to inhibit the proliferation of YD-38 cells were studied at concentrations ranging 


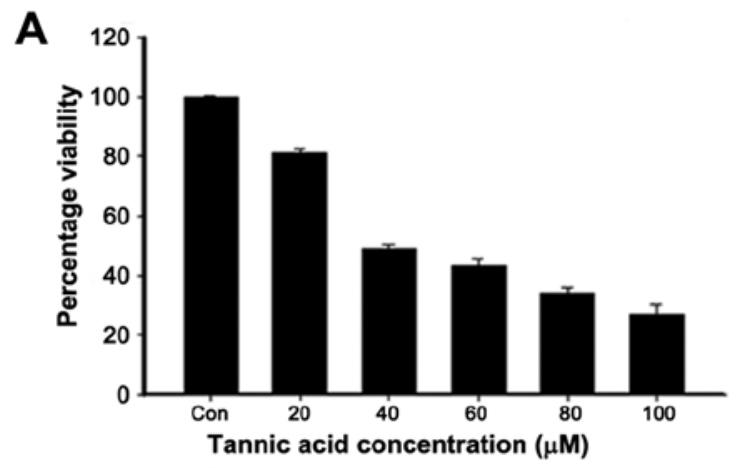

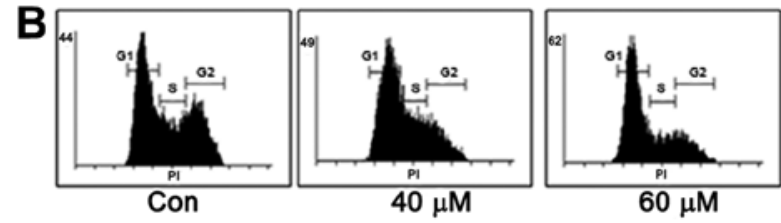

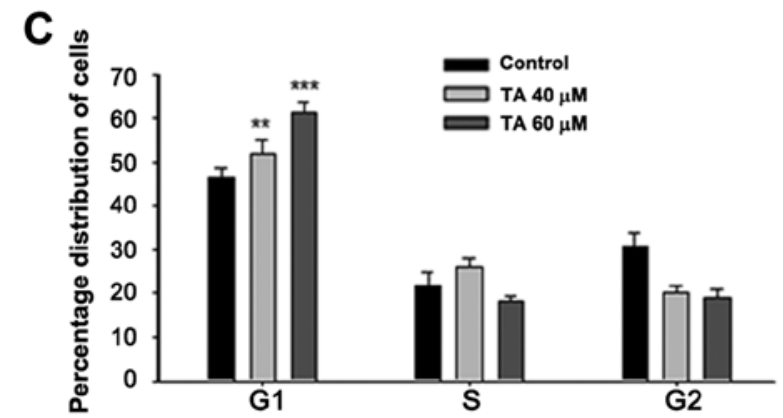

D
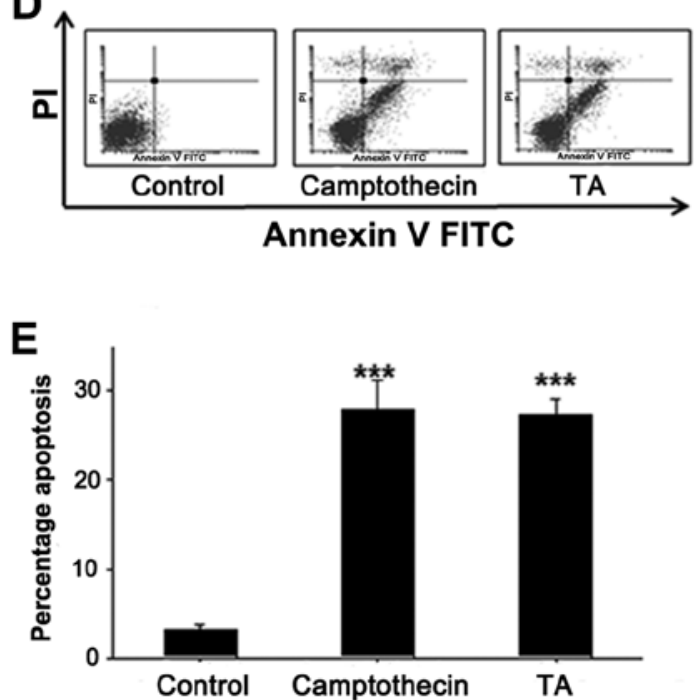

Figure 1. Tannic acid induces apoptosis in gingival cancer cells. (A) Gingival cancer cell lines were treated with increasing concentration of TA for $48 \mathrm{~h}$ and the viability was checked using the crystal violet method. (B) YD-38 cells showing inhibition of cells at G1 phase. (C) Presentation of the cell cycle arrest induced by TA on gingival cancer cells. (D) Annexin V-FITC method showing induction of apoptosis in TA-treated YD-38 cells. (E) The percentage of apoptosis induced is presented as a graph, camptothecin served as positive control. The data are one representative of at least three independent experiments. Asterisks indicate a significant induction of $\mathrm{G} 1$ arrest or apoptosis by ANOVA test $\left({ }^{* *} \mathrm{P}<0.01\right.$; and $\left.{ }^{* * *} \mathrm{P}<0.001\right)$.

from 20 to $100 \mu \mathrm{M}$. TA inhibited the cell viability of YD-38 cells with $\mathrm{IC}_{50}$ values ranging from 50 to $70 \mu \mathrm{M} / 1$ for 48 -h treatment (Fig. 1). The effects of TA on cell viability occurred very slowly. Following 48-h treatment, $60 \mu \mathrm{M}$ TA decreased cell viability by $50 \%$. Analysis of cell viability showed, TA did not induced cell death up to $24 \mathrm{~h}$, rather, it inhibited the proliferation by inducing cell cycle arrest.

Tannic acid induces G1/Sphase arrest in gingival cancer cells. Studies conducted to investigate the role of TA on gingival cancer cell proliferation showed a prominent growth inhibition (Fig. 1). In order to uncover the mechanism of growth arrest, gingival cancer cells were treated with TA at different concentrations (40 and $60 \mu \mathrm{M}$ ). Following TA treatment, the cells were stained using PI and the distribution of nucleous analyzed using FACSCalibur. The study revealed that, in cells treated with $40 \mu \mathrm{M}$ TA, there is an accumulation of cells in the G1 phase with a decrease in percentage of cell population on the $\mathrm{G} 2$ phase (Fig. 1B). In case of $60 \mu \mathrm{M}$ TA treated cells $\left(61 \% ;{ }^{* * *} \mathrm{P}<0.001\right)$, the percentage accumulation of cells in the G1 phase was comparatively higher than that of the $40 \mu \mathrm{M}$ treated cells $\left(51 \%{ }^{* *} \mathrm{P}<0.01\right)$, showing a concentrationdependent G1 phase arrest on the gingival cancer cells
(Fig. 1C). Based on the ability of this concentration to induce cell cycle arrest, the concentration was used for the subsequent experiments to elucidate the signaling events involved in TA mediated G1 arrest.

Tannic acid induces apoptosis in YD-38 cells. We next focused on whether TA has the capacity to induce apoptosis in gingival cancer cells as it does in breast and AML cells (21). The study revealed that, in cells treated with TA, there was an accumulation of cells in the apoptotic phase (Fig. 1D). In case of $60 \mu \mathrm{M}$ TA-treated cells $\left(27.30 \%\right.$, Fig. $\left.1 \mathrm{E} ;{ }^{* * *} \mathrm{P}<0.001\right)$, the percentage of apoptosis was similar to that of the positive control, camptothecin $10 \mu \mathrm{M}$ treated cells $(27.91 \%$, Fig. 1E; $\left.{ }^{* * *} \mathrm{P}<0.001\right)$, confirming induction of apoptosis in the gingival cancer cells.

Tannic acid inhibits the Jak2/STAT3 pathway. The role of STAT3 in the induction of apoptosis was reported previously (15). In our study also, TA inhibited the expression as well as phosphorylation of STAT3 (Fig. 2A). In the cytoplasm, STAT3 is phosphorylated by upstream kinases Jak2. Following phosphorylation it forms homo- or hetero-dimers and translocate to nucleus there it controls transcriptional functions of multiple 
A

$\mathrm{TA}(\mu \mathrm{M})$

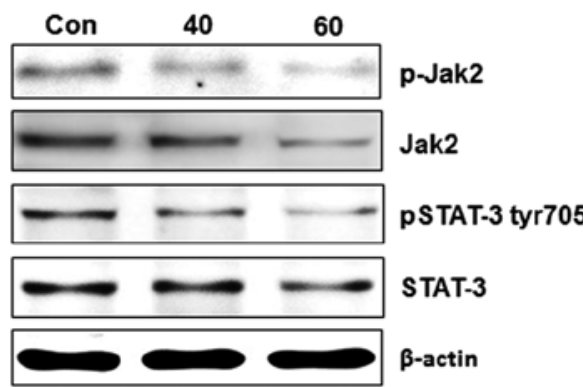

B

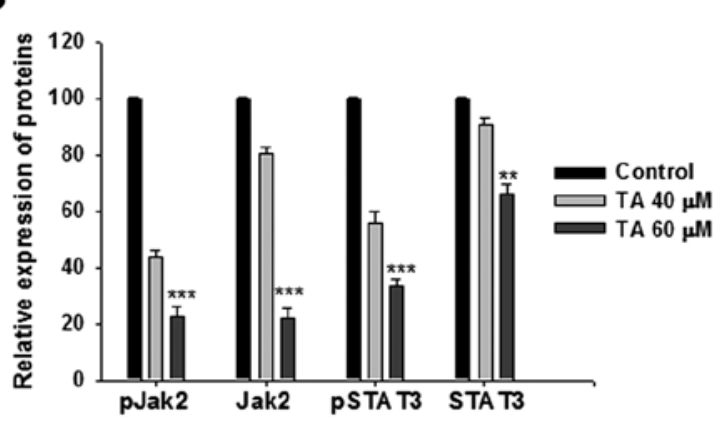

C

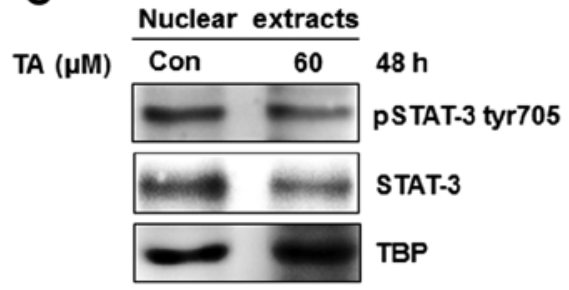

D

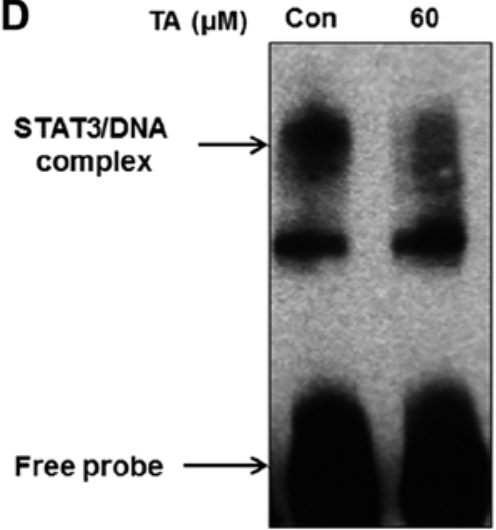

Figure 2. Tannic acid inhibited the Jak2/STAT3 pathway in gingival cancer cells. (A) Gingival cancer cells treated with or without tannic acid for 24 h. Western blotting of whole cell lysate showing inhibition on the expression and phosphorylation of Jak 2 and STAT3 molecules. $\beta$-actin served as loading control. (B) The relative expression of proteins under tannic acid treated conditions. Asterisks indicate a significant inhibition of the expression as well as phosphorylation of Jak2 and STAT3 by ANOVA test $\left({ }^{* *} \mathrm{P}<0.01\right.$; and $\left.{ }^{* * *} \mathrm{P}<0.001\right)$. (C) Western blot analysis of nuclear extracts showing a decrease in nuclear translocation of STAT3 and pSTAT3. TATA binding protein served as the loading control. (D) EMSA analysis showing the decline in DNA binding activities of STAT3. The data are one representative of at least three independent experiments.

genes. Western blot analysis of whole cell extracts showed a reduction in tyr705 phosphorylated STAT3 together with total STAT3. Phosphorylation of STAT3 is primarily relying on Jak2 phosphorylation. Hence the Jak2 (Y1007/Y1008) phosphorylation levels were checked in these cell lines. As expected, TA regulated Jak2 phosphorylation (Fig. 2A). The relative expression analysis showed a concentration-dependent and significant inhibition on the Jak2, STAT3 expression as well as their phosphorylation (Fig. $2 \mathrm{~B} ;{ }^{* *} \mathrm{P}<0.01$ and ${ }^{* * *} \mathrm{P}<0.001$ ).

Tannic acid suppresses the transcriptional functions of STAT3. STAT nuclear translocation and DNA binding activities are influenced by STAT tyrosine phosphorylation rather than serine phosphorylation $(12,13)$. Translocation of initiated STATs to the nucleus follows its binding to a specific response elements in the target gene promoters, and transcriptionally activates the genes. As shown in Fig. 2C, there was a decrease in the nuclear level of pSTAT3 in TA-treated cells, when compared with the control cells. The transcriptional functions of STAT3 is dependent on its ability to bind with specific response element in the target genes. EMSA analysis specific to the STAT3-TF showed a decline in DNA binding activity upon TA treatment (Fig. 2D).

Tannic acid increases the expression of $p 21^{\text {Wafl/Cipl }}$ and $p 27^{\text {Kip }}$. The treatment of gingival cancer cells with TA induced G1 phase arrest. The inhibition of G1/S phase transition is primarily dependent on the $\mathrm{p} 21^{\mathrm{Waf} 1 / \mathrm{Cip} 1}$ and $\mathrm{p} 27^{\mathrm{Kip}}$ levels (22) and its loss leads to uncontrolled cell proliferation. Our studies revealed that, treatment with TA intensified the expression of $\mathrm{p} 21^{\mathrm{Waf} 1 / \mathrm{Cip} 1}$ and $\mathrm{p} 27^{\mathrm{Kip}}$ transcriptionally (Fig. 3A). The elevation in the expression followed a dose-depended pattern and after a period of $24 \mathrm{~h}$ the increase was significant (Fig. 3B; $\left.{ }^{* * *} \mathrm{P}<0.001\right)$. Moreover, the activation of $\mathrm{p} 21^{\mathrm{Waf} 1 / \mathrm{Cip} 1}$ and $\mathrm{p} 27^{\mathrm{Kip}}$ showed a statistically significant pattern.

Inhibition of Jak2/STAT3 pathway suppressed the expression of cyclin D1, cyclin E and CDK-4. Members of cyclins and CDKs are important mediators of the cell cycle. The translational level expression of cyclin D1, cyclin E and CDK-4 were concentration-dependently inhibited by TA (Fig. 3C). The level of CDK-4 was inhibited at protein level (Fig. 3D; $\left.{ }^{* * *} \mathrm{P}<0.001\right)$ but non-significantly inhibited at transcriptional level (Fig. 3B).

Suppression of STAT3/DNA binding activity leads to decline of anti-apoptotic gene products. RT-PCR studies were carried out in YD-38 cells treated with increasing concentrations of TA by random priming of total RNA. Bcl-2 and Bcl- $\mathrm{X}_{\mathrm{L}}$ was amplified using gene specific primers. As TA declined the STAT3 and pSTAT3 levels and their DNA binding activity, it also downregulated the STAT3 target gene products such as Bcl-2, and Bcl-X $\mathrm{L}_{\mathrm{L}}$ (Fig. 4A). By modulating these molecules, TA can target anti-apoptotic mediators and induce apoptosis.

Tannic acid downregulates the anti-apoptotic proteins in concentration-dependent manner. Western blotting studies were carried out in YD-38 cells treated with increasing 


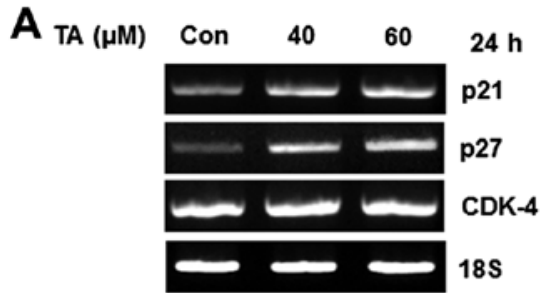

B

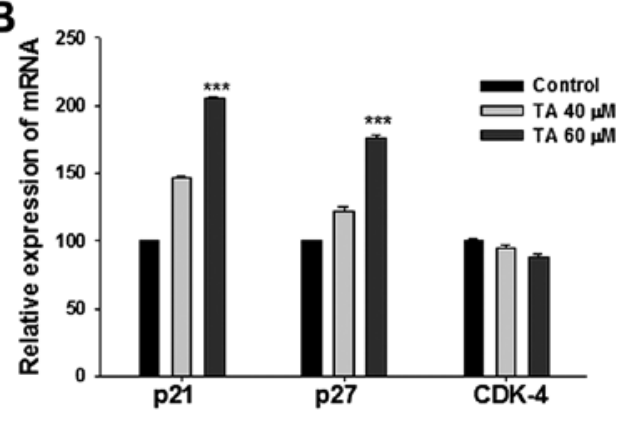

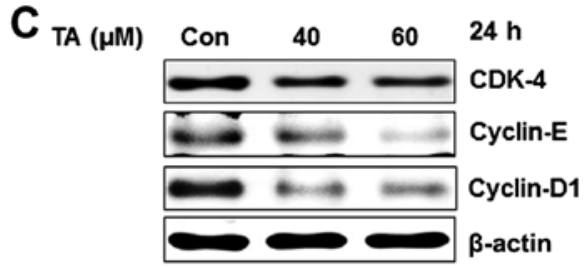

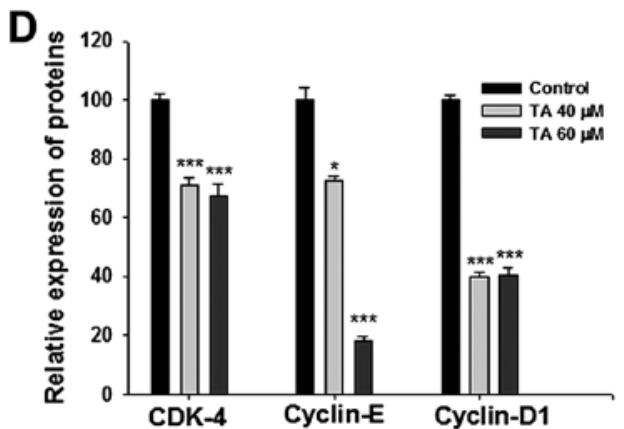

Figure 3. Tannic acid modulates G1 regulators. (A) YD-38 cells were treated with tannic acid for $24 \mathrm{~h}$, the total RNA isolated and subjected for RT-PCR analysis. 18S served as loading control. (B) Relative expression of negative regulators of the cell cycle. The data are presented as the percentage of control and normalized to the loading control 18S. (C) Western blotting of tannic acid treated gingival cancer cells showing inhibition of the positive regulators of cell cycle. $\beta$-actin served as the loading control. (D) Relative expression of the postive regulators of cell cycle. The data are presented as the percentage of control and were normalized to $\beta$-actin. The data are one representative of at least three independent experiments. Asterisks indicate a significant increase or decrease in the transcriptional or translational regulation of positive and negative regulators of the cell cycle by ANOVA test $\left({ }^{* * * *} \mathrm{P}<0.001\right)$.

A

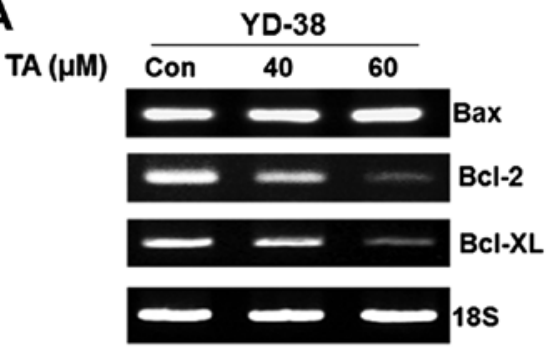

B

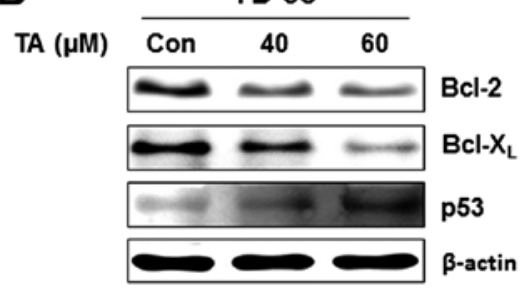

C

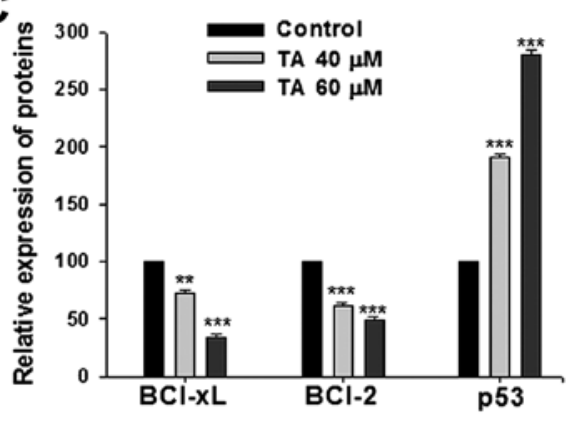

D

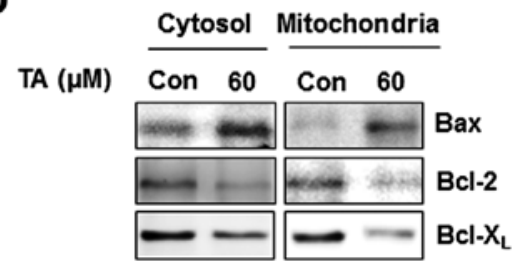

Figure 4. Tannic acid modulates the expression and localization of apoptotic regulators. (A) Total RNA prepared from tannic acid treated and non-treated YD-38 cells were analyzed using RT-PCR. (B) Western blot analysis of apoptotic regulators on the whole cell lysate prepared from gingival cancer cells treated with increasing concentration of tannic acid. (C) Relative expression of apoptotic regulatory proteins. The data are presented as the percentage of control and normalized to $\beta$-actin. Asterisks indicate a significant increase or decrease in the translational regulation of apoptotic regulators by $A N O V A$ test $\left({ }^{* *} \mathrm{P}<0.01\right.$; and ${ }_{* * * *} \mathrm{P}<0.001$ ). (D) Western blot analysis of cytosolic and mitochondrial protein on treatment with tannic acid. The data are one representative of at least three independent experiments.

concentrations of TA. The whole cell lysates were prepared and subjected for the detection of $\mathrm{Bcl}-2$ and $\mathrm{Bcl}-\mathrm{X}_{\mathrm{L}}$. TA exposure led to downregulation of STAT3 target gene products, Bcl-2, and Bcl- $\mathrm{X}_{\mathrm{L}}$ (Fig. 4B). The p53 expression was activated by the treatment with TA (Fig. 4B). The inhibition of anti-apoptotic genes and activation of p53 gene were concentration-dependent and statistically significant (Fig. 4C; ${ }^{* * *} \mathrm{P}<0.001$ ).
Tannic acid inhibits the level of mitochondrial Bcl-2, $B c l-X_{L}$ and increases the mitochondrial localization of Bax. Mitochondria were isolated from the TA-treated and nontreated gingival cancer cells and the cytosol fraction was collected. Western blot analysis of the isolated mitochondria showed inhibition in the level of both Bcl-2 and Bcl- $\mathrm{X}_{\mathrm{L}}$ (Fig. 4D). Increase in mitochondrial localization of Bax was observed with exposure to TA (Fig. 4D) with increased Bax 
A

TA ( $\mu \mathrm{M})$

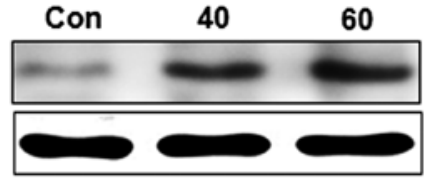

Cyt C $\beta$-actin

B TA ( $\mu$ M)
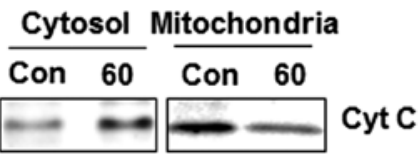

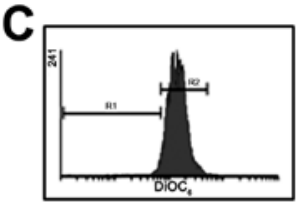

Con

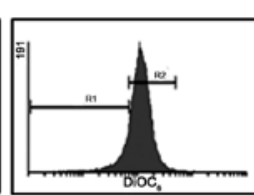

Camptothecin

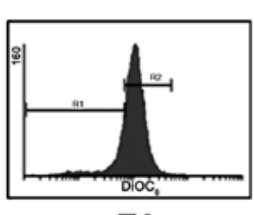

TA

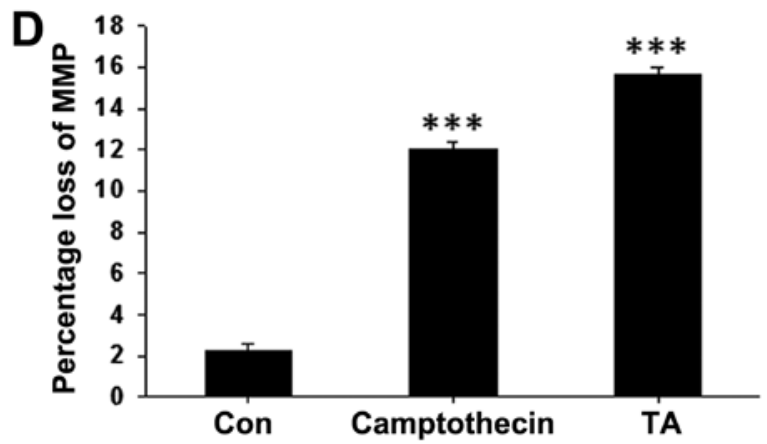

Figure 5. Tannic acid induces loss of mitochondrial membrane potential. (A) Gingival cancer cells were treated with increasing concentration of tannic acid and the whole cell lysates prepared and analyzed. Western blotting showing the concentration-dependent increase in cytochrome $c$ $\beta$-actin served as loading control. (B) Mitochondrial and cytosolic compartmentalization analysis of cytochrome $c$ using western blotting (C) Gingival cancer cells were treated with tannic acid and the loss of mitochondrial membrane potential evaluated using $\mathrm{DiOC}_{6}$ staining followed by FACS analysis. (D) Presentation of the percentage loss of mitochondrial membrane potential. Camptothecin served as a positive control. Asterisks indicate a significant loss in mitochondrial membrane potential using ANOVA test $\left({ }^{* * *} \mathrm{P}<0.001\right)$. The data are one representative of at least three independent experiments.

in the cytosol. Expression levels of Bax also found increased at transcriptional level (Fig. 4A). Similarly, the mitochondrial localization $\mathrm{Bcl}-2$ and $\mathrm{Bcl}-\mathrm{X}_{\mathrm{L}}$ decreased. Whole cell lysates showed decreased mitochondrial pore factors confirming our previous findings of TA on these factors.

Release of cytochrome $c$ to the cytoplasm is observed in tannic acid-treated gingival cancer cells. Whole cell lysates were prepared from TA-treated and non-treated YD-38 cells and subjected for western blot analysis. We found a concentration-dependent increase on the cytochrome $c$ level (Fig. 5A). In order to find the localization of cytochrome $c$ and release of cytochrome $c$ to the cytosol, mitochondrial and cytosolic fractions of YD-38 cells were prepared from TA-treated and non-treated cells and subjected for western blot analysis. In which, we detected a decrease of cytochrome $c$ in mitochondrial fractions and increased levels on the cytosolic fractions (Fig. 5B) indicating the release of cytochrome $c$ to the cytosol and a loss of mitochondrial membrane potential.
Tannic acid induces loss of mitochondrial membrane potential $(\Delta \Psi m)$ in gingival cancer cells. The release of cytochrome $c$ from mitochondria is also usually preceded or accompanied by a reduction in the $\Delta \Psi \mathrm{m}$. To address whether the TA-induced alteration in pore factors were associated with the change of $\Delta \Psi \mathrm{m}$, gingival cancer cells were treated with TA for pre-determined time and stained with $\mathrm{DiOC}_{6}$ to access $\Delta \Psi \mathrm{m}$ (Fig. 5C). Treatment of TA reduced the $\Delta \Psi \mathrm{m}$ significantly (Fig. 5D; $\left.{ }^{* * *} \mathrm{P}<0.001\right)$, showing that the mechanism of apoptosis induction by TA was through the mitochondria-dependent pathway in YD-38 cells.

Caspase activation is required for TA-mediated apoptosis. Mitochondrial apoptotic pathways require active caspases to ensure apoptosis. Whole caspase activation on TA challenged cells were analyzed using poly-caspase assay kit, and showed a prominent increase in caspase activation (Fig. 6A). After observing a significant increase in whole caspase (Fig. 6B), we analyzed the activation of caspase-3. Western blot analysis of TA-treated YD-38 cells showed cleaved form of caspase- 3 confirming the role of caspases in TA-induced apoptosis (Fig. 6C). In-order to confirm the role of caspase-3 in TA- induced apoptosis, cells were treated with the caspase specific inhibitor Z-VAD-FMK prior to TA treatment. The results showed an increase in active caspase- 3 in TA-treated cells comparing to the non-treated control cells (Fig. 6D). Moreover, inhibition of caspase-3 prior to TA treatment inhibited apoptosis and caspase-3 activation significantly (Fig. 6E). These data confirmed that TA induces caspase-dependent apoptosis and activation of caspase- 3 is an essential step for apoptosis induced by TA.

\section{Discussion}

Natural compounds are the possible source of molecules that may have anti-proliferative effects on broad range of cancers. Multiple natural chemicals are being tested for their activities on different forms of cancer. Dietary habits and oral health has direct connection. Foods rich in antioxidants has multiple therapeutic potential on oral health including, prevention from inflammation to malignancies through their bio-active, non-nutrient components $(23,24)$. Conventional treatment modalities such as chemotherapy, radiation, surgery and immunotherapy have shown advantages to various extent in tumor growth retention. However, these modalities can result in problems like speech impairment, cosmetic issues, and face deformities.

Cancer cells are usually reported as uncontrolled cell proliferation occuring as a result of alterations in the positive and negative regulators of the cell cycle. Similarly resistance to apoptotic signals cause prolonged lifespan of cancer cells $(25,26)$. As previously documented, polyphenols and members of tannin family have the capability to induce G1 arrest in various cancer cells. Research is being performed to elucidate the mechanistic aspects of medicinal properties constituted by TA. TA, a glucoside of gallic acid polymer, has been shown to possess anti-bacterial, anti-enzymatic, antitumor and astringent properties. In our study, TA showed proliferation inhibition capability on YD-38 gingival cancer cells (Fig. 1A). 


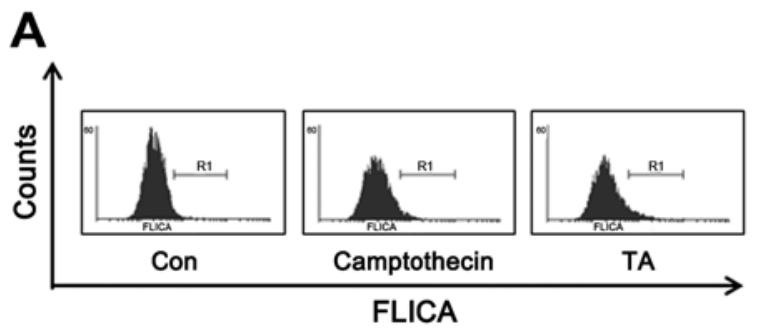

B

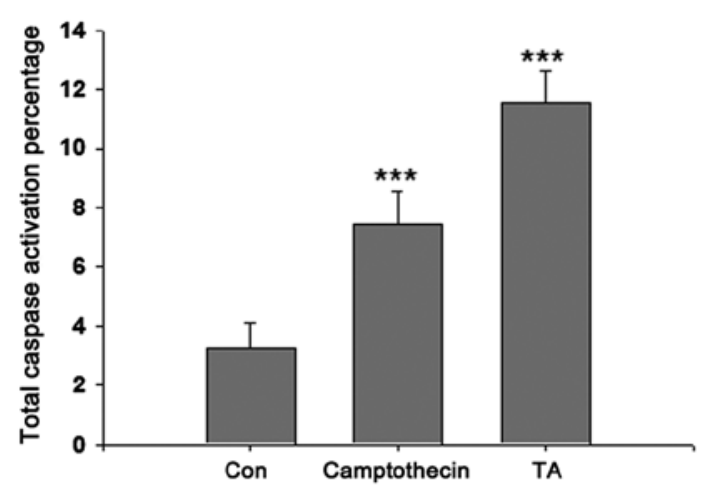

C

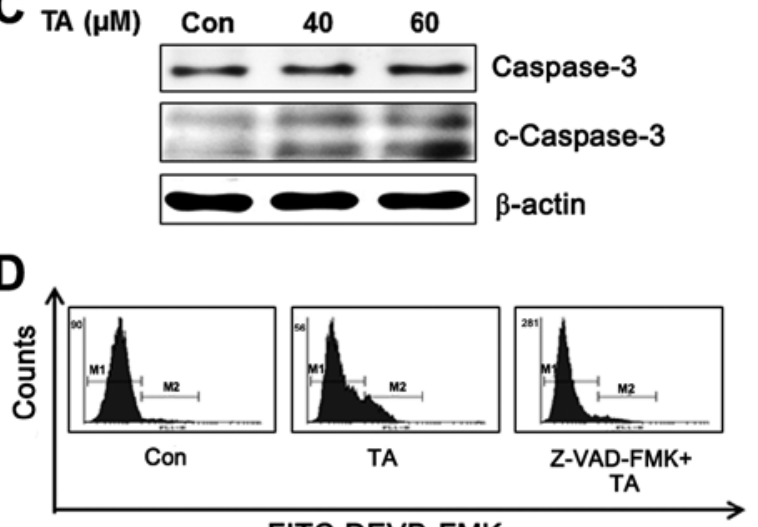

FITC-DEVD-FMK

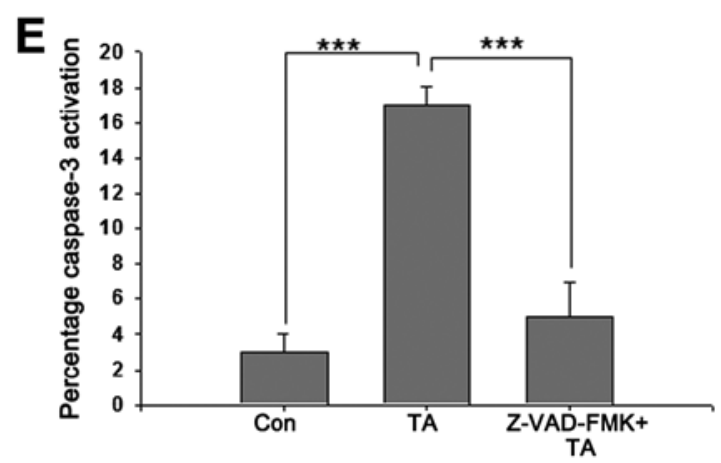

Figure 6. Tannic acid induced caspase-3 activation in gingival cancer cells. (A) Gingival cancer cells were treated with tannic acid and stained with FAM-FLICA poly-caspase stain, then analyzed using FACS. (B) Presentation of the percentage activation of total caspases. Asterisks indicate a significant caspase activation using ANOVA test $\left({ }^{* * *} \mathrm{P}<0.001\right)$. (C) Western blot analysis of gingival cancer cells treated with tannic acid showing the increase in caspase-3 and cleaved caspase-3. (D) Gingival cancer cells were pre-treated or non-treated with caspase-3 inhibitor followed by treatment with tannic acid. The cells then stained with FITC-DEVD-FMK for $1 \mathrm{~h}$ and analyzed using FACS. (E) Presentation of the percentage activation of caspase-3. Asterisks indicate a significant activation of caspase-3 using t-test $\left({ }^{* * *} \mathrm{P}<0.001\right)$. The data are one representative of at least three independent experiments.

Escape from normal apoptotic pathways is a common phenomenon found in almost all types of cancers. Hence, making the cells susceptible to apoptosis is a principal approach for developing drugs against malignancies. Different cytotoxic agents proved their efficiency in inducing apoptosis and are being used as chemotherapeutics for the treatment for various human malignancies. Even though they are effective to an extent, their toxic effect is associated with side effects. Screening of multiple agents is taking place to find effective chemotherapeutic agents with the ability to control cell proliferation without side effects. TA has been shown to have anticancer properties by inducing apoptosis and controlling the cancer cell proliferation $(21,27)$. Previous studies suggest that TA has properties such as the inhibition of CXCL12 (SDF-1 $\alpha$ )/ and CXCR4 (28).

In the present study, TA inhibited the phosphorylation of Jak2 (Fig. 2A). Jak2 is the major upstream regulator of STAT3 phosphorylation (29). Inhibition of Jak2 phosphorylation resulted in inhibition of STAT3 phosphorylation. Generally, STAT3 is phosphorylated on S727 and Y705 residues. Thus, Y705 is responsible for the nuclear translocation and DNA binding activities of STAT3 (30). TA inhibited the phosphorylation of Y705 residues in STAT3. Analysis of nuclear extracts also confirmed the inhibition of STAT3 nuclear translocation. Gel shift analysis showed that the DNA binding activity of STAT3 also inhibited by TA treatment (Fig. 2D). One of the major functions of STAT3 is to bind to its downstream target genes and transcriptionally activate them. Transcriptional analysis of STAT3 downstream targets such as cyclin D1, Bcl-2, and Bcl- $\mathrm{X}_{\mathrm{L}}$ confirmed the ability of TA in inhibiting transcriptional activation of STAT3. These targets are directly connected with cell cycle arrest as well as apoptosis, showing the connection between inhibition of STAT3 and induction of proliferation regulation.

In nearly all mammalian cells, proliferation is mainly controlled in G1 phase and it automatically progress through the remaining phases (31). It is reported that, G1 arrest is p53-dependent (32). In support of this, our study also showed an increase in the translational level of p53. Most of the anticancer agents induced G1 arrest through decreasing the activity of CDKs and increasing the expression of CKIs (33-35). In the present study, using TA an inhibition on the positive regulators of cell cycle, cyclin D1, cyclin E and CDK-4 were shown. Moreover, it transcriptionally activated the negative regulators of the cell cycle, $\mathrm{p} 21^{\text {Waf } 1 / \text { Cip } 1}$ and p2 $7^{\text {Kipl }}$. Which induced a prominent G1 arrest in TA-treated YD-38 cells (Fig. 1B).

Apoptosis occurs through different mechanisms, in the extrinsic pathway; an external signal stimulates the apoptotic cascade and in the intrinsic pathway, intracellular factors trigger the apoptotic cascade (36). The intrinsic pathway is usually under the control of the mitochondria, and is also known as mitochondrial pathway. The role of $\mathrm{Bcl}-2$ and $\mathrm{Bcl}-\mathrm{X}_{\mathrm{L}}$ on TA induced apoptosis was confirmed by analysis of mito- 
chondrial protein levels. In mitochondria, Bcl-2 and Bcl- $\mathrm{X}_{\mathrm{L}}$ act as anti-pore factors and inhibit the release of cytochrome $c$ to the cytosol and inhibit apoptosis $(37,38)$. Mitochondria isolated from TA-treated cells showed a reduction on both Bcl-2 and $\mathrm{Bcl}-\mathrm{X}_{\mathrm{L}}$ pointing to the loss of pore closing factors. It was previously reported that TA has the capacity to increase the expression of Bax (39). In our study we observed that, Bax is highly expressed and localized on the mitochondria. Changes in the localization of mitochondrial pore and anti-pore factors lead to the loss of mitochondrial membrane potential $(\Delta \Psi \mathrm{m})$. Any alteration in $\Delta \Psi \mathrm{m}$ leads to the activation of mitochondrial apoptotic pathway through the release of cytochrome $c$ to the cytosol (Fig. 5).

Cytochrome $c$ is an activator of zymogenic caspases. Once the cytochrome $c$ is released to the cytosol, it activates the pro-caspase to active caspase (40). Fig. 6A shows the activation of poly caspases. Apoptosis is usually carried out with the activation of the effector caspase-3. Western blot analysis of TA-treated cells also show the cleaved forms of caspase-3 (Fig. 6B). Inhibition of caspase-3 using a specific inhibitor showed a significant recovery from TA-induced apoptosis (Fig. 6E). This confirmed the caspase-dependent mitochondrial pathway in the TA mediated apoptosis.

According to the present study, TA induces G1 arrest and apoptosis in human gingival cancer cells. The mechanistic aspects of TA mediated apoptosis depend primarily on the inhibition of the Jak2/STAT3 pathway. Here we report that the Jak2/STAT3 pathway is involved in the cell cycle arrest and the intrinsic (mitochondrial) apoptotic pathway. We suggest the use of TA and other drugs targeting STAT3 as a trial drug for inducing G1 arrest and intrinsic apoptosis.

\section{Acknowledgements}

This study was supported by Konkuk University in 2014 .

\section{References}

1. Gomez D, Faucher A, Picot V, Siberchicot F, Renaud-Salis JL, Bussières $\mathrm{E}$ and Pinsolle $\mathrm{J}$ : Outcome of squamous cell carcinoma of the gingiva: a follow-up study of 83 cases. J Craniomaxillofac Surg 28: 331-335, 2000

2. Yokoo S, Umeda M, Komatsubara H, Shibuya Y and Komori T: Evaluation of T-classifications of upper gingival and hard palate carcinomas - a proposition for new criterion of T4. Oral Oncol 38: 378-382, 2002.

3. Torabinejad M and Rick GM: Squamous cell carcinoma of the gingiva. J Am Dent Assoc 100: 870-872, 1980

4. Pathak KA, Mathur N, Talole S, Deshpande MS, Chaturvedi P, Pai PS, Chaukar DA and D'Cruz AK: Squamous cell carcinoma of the superior gingival-buccal complex. Oral Oncol 43: 774-779, 2007.

5. Bian Y, Masuda A, Matsuura T, Ito M, Okushin K, Engel AG and Ohno K: Tannic acid facilitates expression of the polypyrimidine tract binding protein and alleviates deleterious inclusion of CHRNA1 exon P3A due to an hnRNP H-disrupting mutation in congenital myasthenic syndrome. Hum Mol Genet 18: 1229-1237, 2009.

6. Naus PJ, Henson R, Bleeker G, Wehbe H, Meng F and Patel T: Tannic acid synergizes the cytotoxicity of chemotherapeutic drugs in human cholangiocarcinoma by modulating drug efflux pathways. J Hepatol 46: 222-229, 2007.

7. Koide T, Kamei H, Hashimoto Y, Kojima T and Hasegawa M: Tannic acid raises survival rate of mice bearing syngeneic tumors. Cancer Biother Radiopharm 14: 231-234, 1999.

8. Gali-Muhtasib HU, Yamout SZ and Sidani MM: Tannins protect against skin tumor promotion induced by ultraviolet-B radiation in hairless mice. Nutr Cancer 37: 73-77, 2000.
9. Nepka C, Sivridis E, Antonoglou O, Kortsaris A, Georgellis A, Taitzoglou I, Hytiroglou P, Papadimitriou C, Zintzaras I and Kouretas D: Chemopreventive activity of very low dose dietary tannic acid administration in hepatoma bearing $\mathrm{C} 3 \mathrm{H}$ male mice. Cancer Lett 141: 57-62, 1999.

10. Gali HU, Perchellet EM and Perchellet JP: Inhibition of tumor promoter-induced ornithine decarboxylase activity by tannic acid and other polyphenols in mouse epidermis in vivo. Cancer Res 51: 2820-2825, 1991.

11. Tikoo K, Bhatt DK, Gaikwad AB, Sharma V and Kabra DG: Differential effects of tannic acid on cisplatin induced nephrotoxicity in rats. FEBS Lett 581: 2027-2035, 2007.

12. Garcia R, Bowman TL, Niu G, Yu H, Minton S, Muro-Cacho CA, Cox CE, Falcone R, Fairclough R, Parsons S, et al: Constitutive activation of Stat 3 by the Src and JAK tyrosine kinases participates in growth regulation of human breast carcinoma cells Oncogene 20: 2499-2513, 2001.

13. Song JI and Grandis JR: STAT signaling in head and neck cancer. Oncogene 19: 2489-2495, 2000.

14. Pedranzini L, Leitch A and Bromberg J: Stat3 is required for the development of skin cancer. J Clin Invest 114: 619-622, 2004.

15. Burke WM, Jin X, Lin HJ, Huang M, Liu R, Reynolds RK and Lin J: Inhibition of constitutively active Stat 3 suppresses growth of human ovarian and breast cancer cells. Oncogene 20: 7925-7934, 2001.

16. Schaefer LK, Ren Z, Fuller GN and Schaefer TS: Constitutive activation of Stat $3 \alpha$ in brain tumors: Localization to tumor endothelial cells and activation by the endothelial tyrosine kinase receptor (VEGFR-2). Oncogene 21: 2058-2065, 2002.

17. Lin J, Tang H, Jin X, Jia G and Hsieh JT: p53 regulates Stat3 phosphorylation and DNA binding activity in human prostate cancer cells expressing constitutively active Stat3. Oncogene 21: 3082-3088, 2002.

18. Park JH, Darvin P, Lim EJ, Joung YH, Hong DY, Park EU, Park SH, Choi SK, Moon ES, Cho BW, et al: Hwanggeumchal sorghum induces cell cycle arrest, and suppresses tumor growth and metastasis through Jak2/STAT pathways in breast cancer xenografts. PLoS One 7: e40531, 2012.

19. Lim EJ, Hong DY, Park JH, Joung YH, Darvin P, Kim SY, Na YM, Hwang TS, Ye SK, Moon ES, et al: Methylsulfonylmethane suppresses breast cancer growth by down-regulating STAT3 and STAT5b pathways. PLoS One 7: e33361, 2012.

20. Yang EB, Wei L, Zhang K, Chen YZ and Chen WN: Tannic acid, a potent inhibitor of epidermal growth factor receptor tyrosine kinase. J Biochem 139: 495-502, 2006.

21. Chen K-S, Hsiao Y-C, Kuo D-Y, Chou MC, Chu SC, Hsieh YS and Lin TH: Tannic acid-induced apoptosis and -enhanced sensitivity to arsenic trioxide in human leukemia HL-60 cells. Leuk Res 33: 297-307, 2009

22. Pavletich NP: Mechanisms of cyclin-dependent kinase regulation: Structures of Cdks, their cyclin activators, and Cip and INK4 inhibitors. J Mol Biol 287: 821-828, 1999.

23. Liu RH: Potential synergy of phytochemicals in cancer prevention: Mechanism of action. J Nutr 134 (Suppl): S3479-S3485, 2004.

24. Venugopal R and Liu RH: Phytochemicals in diets for breast cancer prevention: The importance of resveratrol and ursolic acid. Food Sci Hum Wellness 1: 1-13, 2012.

25. Hanahan D and Weinberg RA: The hallmarks of cancer. Cell 100: 57-70, 2000 .

26. Hanahan D and Weinberg RA: Hallmarks of cancer: The next generation. Cell 144: 646-674, 2011.

27. Cosan D1, Soyocak A, Basaran A, Degirmenci I and Gunes HV: The effects of resveratrol and tannic acid on apoptosis in colon adenocarcinoma cell line. Saudi Med J 30: 191-195, 2009.

28. Chen X, Beutler JA, McCloud TG, Loehfelm A, Yang L, Dong HF, Chertov OY, Salcedo R, Oppenheim JJ and Howard OM: Tannic acid is an inhibitor of CXCL12 (SDF-1 $\alpha$ )/CXCR4 with antiangiogenic activity. Clin Cancer Res 9: 3115-3123, 2003.

29. Imada K and Leonard WJ: The Jak-STAT pathway. Mol Immunol 37: 1-11, 2000.

30. Bromberg JF, Wrzeszczynska MH, Devgan G, Zhao Y, Pestell RG, Albanese C and Darnell JE Jr: Stat 3 as an oncogene. Cell 98: 295-303, 1999.

31. Pardee AB: G1 events and regulation of cell proliferation. Science 246: 603-608, 1989.

32. Wang X, Bai H, Zhang X, Liu J, Cao P, Liao N, Zhang W, Wang Z and Hai C: Inhibitory effect of oleanolic acid on hepatocellular carcinoma via ERK-p53-mediated cell cycle arrest and mitochondrial-dependent apoptosis. Carcinogenesis 34: 1323-1330, 2013. 
33. Hong $\mathrm{C}$, Kim H-A, Firestone GL and Bjeldanes LF: 3,3'-Diindolylmethane (DIM) induces a G (1) cell cycle arrest in human breast cancer cells that is accompanied by Sp1-mediated activation of p21 (WAF1/CIP1) expression. Carcinogenesis 23: 1297-1305, 2002.

34. Yokota T, Matsuzaki Y, Koyama M, Hitomi T, Kawanaka M, Enoki-Konishi M, Okuyama Y, Takayasu J, Nishino H, Nishikawa A, et al: Sesamin, a lignan of sesame, down-regulates cyclin D1 protein expression in human tumor cells. Cancer Sci 98: 1447-1453, 2007.

35. Garcia HH, Brar GA, Nguyen DHH, Bjeldanes LF and Firestone GL: Indole-3-carbinol (I3C) inhibits cyclin-dependent kinase-2 function in human breast cancer cells by regulating the size distribution, associated cyclin $\mathrm{E}$ forms, and subcellular localization of the CDK2 protein complex. J Biol Chem 280: 8756-8764, 2005.
36. Elmore S: Apoptosis: A review of programmed cell death. Toxicol Pathol 35: 495-516, 2007.

37. Gross A, McDonnell JM and Korsmeyer SJ: BCL-2 family members and the mitochondria in apoptosis. Genes Dev 13: 1899-1911, 1999.

38. Chen YB, Aon MA, Hsu Y-T, Soane L, Teng X, McCaffery JM, Cheng WC, Qi B, Li H, Alavian KN, et al: $\mathrm{Bcl}_{\mathrm{L}}$ regulates mitochondrial energetics by stabilizing the inner membrane potential. J Cell Biol 195: 263-276, 2011.

39. Nam S, Smith DM and Dou QP: Tannic acid potently inhibits tumor cell proteasome activity, increases p27 and Bax expression, and induces G1 arrest and apoptosis. Cancer Epidemiol Biomarkers Prev 10: 1083-1088, 2001.

40. Jiang $X$ and Wang X: Cytochrome $c$ promotes caspase-9 activation by inducing nucleotide binding to Apaf-1. J Biol Chem 275: 31199-31203, 2000. 